\title{
Combination Restoration for Motion-blurred Color Videos under Limited Transmission Bandwidth
}

\author{
Shi Li, Yuping Feng \\ Changchun Institute of Optics, Fine Mechanics and Physics, \\ Chinese Academy of Sciences, Changchun 130033, China; \\ Graduate University of the Chinese Academy of Sciences, Beijing 100039, China \\ E-mail:brightlishi@gmail.com
}

Bao Zhang, Hui Sun

Changchun Institute of Optics, Fine Mechanics and Physics, Chinese Academy of Sciences, Changchun 130033, China;

\begin{abstract}
Color video images degraded in a deterministic way by motion-blurring can be restored by the new algorithm in real-time by using color components combination to fit to the limited transmission bandwidth. The image motion PSF of each surface of YUV422 image can be obtained based on the color space conversion model. The $Y, U$, $\mathrm{V}$ planes are packed to construct a 2 dimensional complex array. Through the decomposition of frequency domain, the $Y, U, V$ frequency can be had respectively by performing Fourier transform a time on the specific complex array. The resulting frequencies will be filtered by Wiener filter to generate the final restored images. The proposed algorithm can restore 1024x1024 24-bit motionblurred color video images at $18 \mathrm{~ms} /$ frame speed on GPU, and the PSNR of the restored frame is 31.45. The experiment results show that the proposed algorithm is $3 \mathrm{X}$ speed compared to the traditional algorithm, and it reduces the bandwidth of video data stream 1/3.
\end{abstract}

Index Terms-motion-blurred; color image; image restoration; real-time computing; graphic processing unit

\section{INTRODUCTION}

Color is described by color space, a mathematical representation of a set of colors. Several color spaces is in wide use, including Hue Saturation Intensity (HSI),

Manuscript received January 19, 2009; revised June 17, 2009; accepted July 21, 2009.
YUV and $\mathrm{RGB}^{[1]}$. For computer applications it is most natural to describe colors in terms of RGB color space $^{[2]}$. In RGB space, color is considered to be an additive mixture of three primary colors: red, green, and blue. RGB space corresponds well to the actual physical devices used to produce colors with computers, such as TV monitors, with red, green, and blue phosphors. Obviously, color image contains more information compared to gray level image and is widely applied in the area of aerial imaging.

Aerial color image is inevitably confused by aerial motion blurred. The restoration of aerial blurred images has been widely studied, and many approaches ${ }^{[3,4,5]}$ based on variation or stochastic formulations tried to solve this ill-posed problem. However, such approaches are mostly designed for gray level images. Generally, the color image should be separated into red, green, and blue images, and then each image is handled separately as a black and white image by those approaches. The obvious defect of this method is that the computational cost is $3 \mathrm{X}$ than the gray level image. There are few approaches, such as vector image restoration $^{[6]}$, exist for multichannel color images. But the computational requirements of them are still huge and do not obviously decrease. 
In the family of television transmission color spaces, we notice that YUV color space becomes a widely used model in digital video processing for the reduction of storage and computation ${ }^{[7]}$. The YUV color space separates RGB into luminance and chrominance information ${ }^{[8]}$. It encodes a color image or video taking human perception into account to reduce bandwidth for chrominance components. In section 2 we propose the method of using YUV to restore the RGB motion blur. In section 3 we introduce the necessity of using YUV color space to replace RGB color space under limited transmission bandwidth. In section 4 and 5 we derive the specific combination of $\mathrm{Y}, \mathrm{U}, \mathrm{V}$ signal which ensures a very efficient fast Fourier transform. Finally, some examples are shown.

\section{MOTION BLURRED OF YUV IMAGES}

The image degradation caused by image motion is often represented in terms of a matrix-vector formation of $(1)^{[4]}$. This is given by

$$
g=f \cdot H
$$

where g, $f$ are the blurred, original images, and $\mathrm{H}$ represent the blur operator. The solutions of (1) were talked about in many books and papers ${ }^{[3,4]}$. Here, let's put the focus on fast restoration using traditional Wiener filter ${ }^{[5]}$.

When the matrices of $\mathrm{g}$ and $f$ are unwrapped, equation (1) can be rewritten as

$$
\begin{aligned}
& {[g(0) g(1) \cdots g(N-1)]} \\
& \quad=[f(0) f(1) \cdots f(N-1)] \cdot H
\end{aligned}
$$

Generally, the original signal from the color image sensor is RGB format. If $\mathrm{g}$ and $f$ are the color images with RGB format, the degradation of the image can be represented by

$$
\begin{aligned}
& {\left[\begin{array}{l}
g_{R}(0) g_{R}(1) \cdots g_{R}(N-1) \\
g_{G}(0) g_{G}(1) \cdots g_{G}(N-1) \\
g_{B}(0) g_{B}(1) \cdots g_{B}(N-1)
\end{array}\right]} \\
& \quad=\left[\begin{array}{l}
f_{R}(0) f_{R}(1) \cdots f_{R}(N-1) \\
f_{G}(0) f_{G}(1) \cdots f_{G}(N-1) \\
f_{B}(0) f_{B}(1) \cdots f_{B}(N-1)
\end{array}\right] \cdot H
\end{aligned}
$$

Equation (2) is considered as the expression of the degradation of the gray level images. According to (2) and (3), if the size of the RGB image and the gray image is the same, it is obviously that the calculated amount of the restoration of RGB images will be $3 \mathrm{X}$ larger than the gray level images.

YUV uses RGB information, but it creates a black and white image from the full color image and then subtracts three primary colors resulting in two additional signals to describe color. The three signals can be combined back together to generate the full color image. Equation (4) reveals the relationship between RGB and YUV.

$$
\begin{aligned}
& {\left[\begin{array}{l}
g_{R}(0) g_{R}(1) \cdots g_{R}(N-1) \\
g_{G}(0) g_{G}(1) \cdots g_{G}(N-1) \\
g_{B}(0) g_{B}(1) \cdots g_{B}(N-1)
\end{array}\right]} \\
& =C \cdot\left[\begin{array}{l}
g_{Y}(0) g_{Y}(1) \cdots g_{Y}(N-1) \\
g_{U}(0) g_{U}(1) \cdots g_{U}(N-1) \\
g_{V}(0) g_{V}(1) \cdots g_{V}(N-1)
\end{array}\right]
\end{aligned}
$$

Where $\mathrm{C}$ is the conversion coefficient matrix of color space. Based on (4), the images can be converted from RGB color space to YUV color space or YUV color space to RGB color space. Using the color conversion and removing $\mathrm{C}$ matrix from both sides of the equation, the degradation of the color image in YUV color space can be given by

$$
\begin{gathered}
{\left[\begin{array}{c}
g_{Y}(0) g_{Y}(1) \cdots g_{Y}(N-1) \\
g_{U}(0) g_{U}(1) \cdots g_{U}(N-1) \\
g_{V}(0) g_{V}(1) \cdots g_{V}(N-1)
\end{array}\right]} \\
\quad=\left[\begin{array}{c}
f_{Y}(0) f_{Y}(1) \cdots f_{Y}(N-1) \\
f_{U}(0) f_{U}(1) \cdots f_{U}(N-1) \\
f_{V}(0) f_{V}(1) \cdots f_{V}(N-1)
\end{array}\right] \cdot H
\end{gathered}
$$

\section{NECESSITY OF USING YUV}

There are two basic kinds of cameras: interlaced scan and progressive scan. Interlaced scan cameras records only half of the horizontal lines at a time (the first field, containing the odd-numbered lines, followed by the second field, containing the even-numbered lines). Hence, an image has to be divided into two fields to be recorded at different time by interlaced scan cameras, and there exists some time delay between two fields that make interfield twitter. That means the 
interlaced scan makes the image motion more complicate, and more difficult to be restored. Progressive scan cameras record all the horizontal lines of a picture at one time as a single frame. Frame format (progressive) is familiar to our image knowledge. To grab color video in way of progressive scan, we find the 3-CCD camera is our best choice.

For easier analysis, we assume that the camera can capture 25 frames (1024x1024x24bit) in 1 second, and the data flow velocity is $75 \mathrm{MB} / \mathrm{s}$. The bandwidth description of current Personal Computer is shown in Fig.1. As can be seen that the bandwidths around north bridge chip are big enough for our data throughput, but the bandwidths of PCI bus and SATA will be the bottleneck of the data flow to block the data throughput.

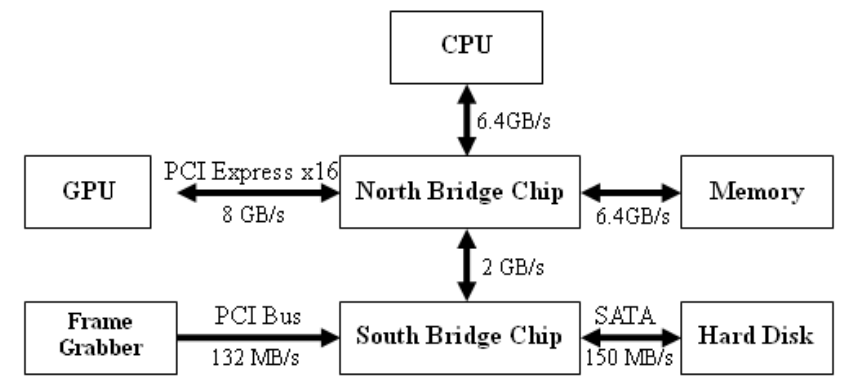

Fig.1 the bandwidth description of currently Personal Computer

We know that image processing program reads next video frame only if the current frame has been totally processed. The Pal format video's frame rate is $25 \mathrm{fps}$. Therefore, the image processing of every frame should be finished in $40 \mathrm{~ms}$ to receive the real-time processing. Fig.2 shows the certain time interval between two frames. During the video processing, we assume the time cost in frame transfer and feedback is equal, we can get the expression of frame transfer time (T2):

$$
T 2=\frac{40 m s-T 3-T 1-T 5}{2}
$$

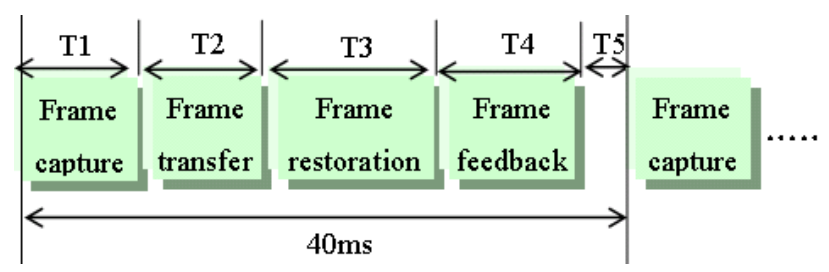

Fig. 2 time chart of image processing

To be simplification, we avoid the frame capture time (T1) and cushion time (T5), and let $\mathrm{T} 1$ and $\mathrm{T} 5$ be zero.
Then, we get the maximum function of frame transfer time (T2):

$$
T 2_{\max }=\frac{40 m s-T 3}{2}
$$

That means the time cost in transfer can't larger than $\mathrm{T} 2{ }_{\max }$ for the real time processing. When the size of the frame data is $\mathrm{S}$, the transfer bandwidth $f$ can be expressed as following:

$$
f=\frac{S}{T 2} \geq \frac{S}{T 2_{\max }}=\frac{2 S}{40 m s-T 3}
$$

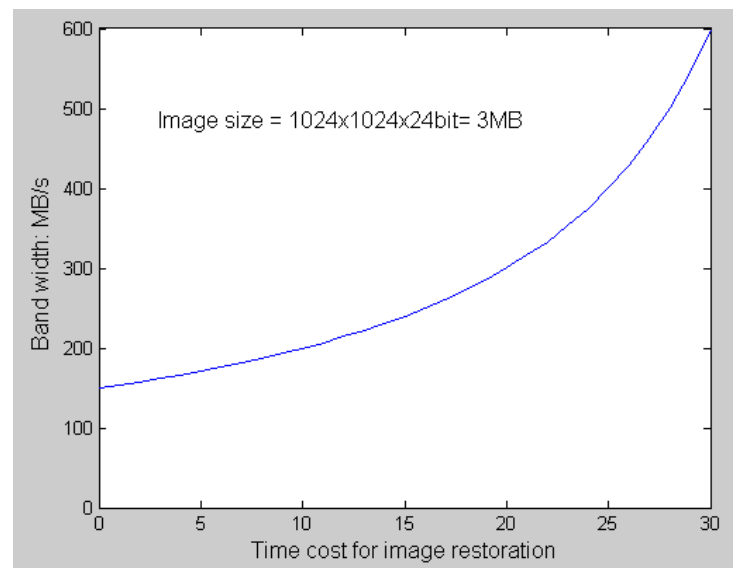

Fig.3 bandwidth requirement analysis of $1024 \times 1024$ 24bit images

According to (8), the relation ship between data transfer bandwidth $f$ and the frame restoration time T3 can be plotted as Fig.3. It is easy to find that even if the single frame, $1024 \times 1024$ 24bit, can be processed in 10 ms, the transfer bandwidth need to be $200 \mathrm{MB} / \mathrm{s}$, which is far larger than $133 \mathrm{MB} / \mathrm{s}$, the theoretical bandwidth of PCI bus, to realize the real time process. Hence, using YUV color space to reduce the bandwidth is meaningful.

\section{YUV CHANNELS COMBINATION}

YUV has an important advantage over RGB, that is the $\mathrm{U}$ and $\mathrm{V}$ components can be represented with $\mathrm{a}$ lower resolution than $\mathrm{Y}$, because the human visual system (HVS) is less sensitive to color than to luminance (brightness). There are several YUV sampling formats, such as 4:4:4, 4:2:2, 4:2:0 sampling. Here, we would like to employ YUV 4:2:2 sampling to realize the approach optimization.

4.1 Motion blurred in YUV 4:2:2 sampling 


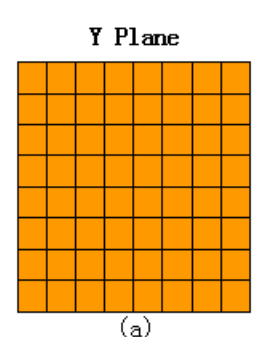

Fig.4 Il

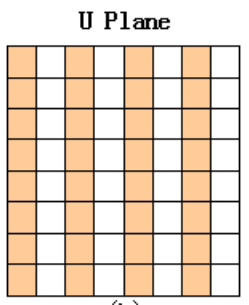

(b)

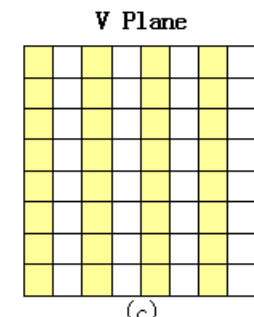

(c)

The image data are archived as planar format, and the YUV 4:2:2 sampling is illustrated in Fig.4. Fig.4-a is $\mathrm{Y}$ plane, and the Fig.4-b, c are U, V plane with lower resolution respectively. In Fig.4-b (and c), the blank pixels are not actually present on the plane, the existence of the blank pixels is just for assistant illustration. Here, make an assumption that all the blank pixels have the same value as their adjacent left pixels respectively. Then, the image motion on the $\mathrm{Y}, \mathrm{U}, \mathrm{V}$ plane will be the same. However, half pixels are over lap in the horizontal component of the image motion on $\mathrm{U}$ (and V) plane for the existence of blank pixels. Sample the $\mathrm{U}, \mathrm{V}$ plane by removing the blank pixels back to the original YUV 4:2:2 format, we can find that the horizontal component of the image motion on $U$ (and V) plane is half of the one on the $\mathrm{Y}$ plane and the vertical component of the image motion on $U$ (and $V$ ) plane is equal to the one on the $\mathrm{Y}$ plane. The specific point spread function (PSF) of the image motion is given by

$$
\begin{aligned}
& h_{U}{ }^{x}(x)=h_{V}{ }^{x}(x)=\frac{1}{2} h_{Y}^{x}(x) \\
& =\left\{\begin{array}{cc}
\frac{2}{L_{Y}{ }^{x}}, & x=0,1 \cdots L_{Y}{ }^{x} / 2-1 \\
0, & \text { else }
\end{array}\right. \\
& h_{U}^{y}(x)=h_{V}^{y}(x)=h_{Y}^{y}(x) \\
& =\left\{\begin{array}{cc}
\frac{1}{L_{Y}{ }^{y}}, x=0,1 \cdots L_{Y}{ }^{y}-1 \\
0, & \text { else }
\end{array}\right.
\end{aligned}
$$

where the superscript $\mathrm{x}, \mathrm{y}$ denote the horizontal and vertical component respectively, and the subscript $\mathrm{Y}, \mathrm{U}$, $\mathrm{V}$ indicate the corresponding plane. $\boldsymbol{L}_{\boldsymbol{Y}}{ }^{x}$ and $\boldsymbol{L}_{\boldsymbol{Y}}{ }^{y}$ stand for the horizontal and vertical component of the image motion on the Y plane respectively.

\subsection{Combination of U, V plane}

A part of chrominance components which is not necessary is reduced by sampling, but there still have three separated image planes, one big and two smalls, needed to be filtered. Equation (9) reveals that U, V plane have the same PSF. And both the two small separated planes, U and V plane, in YUV 4:2:2 format have the half size of $Y$ plane. For more efficiency, the $U$, $\mathrm{V}$ plane can be combined to form a new plane, UV plane, which has the same size as $\mathrm{Y}$ plane. Of course, the PSF of the UV plane is the same as the single $U$ (or $\mathrm{V})$ plane.

In order to explain the combination more intuitively, the resulted images combination are shown in Fig.5 and Fig.6. Fig.5-a, b are the Y, UV plane of the blurred "Lena” image respectively. The PSF of image motion of them are described in (9). According to the blurred PSFs, the blurred $\mathrm{Y}, \mathrm{UV}$ plane can be restored by Wiener filter ${ }^{[3,4,5]}$ respectively. The restored $\mathrm{Y}$, UV plane are shown in Fig.6. Both the two groups' planes can be combined back together to result their corresponding full color image.

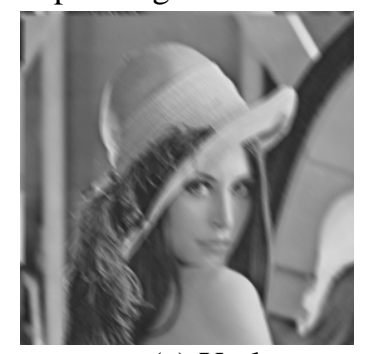

(a) Y plane

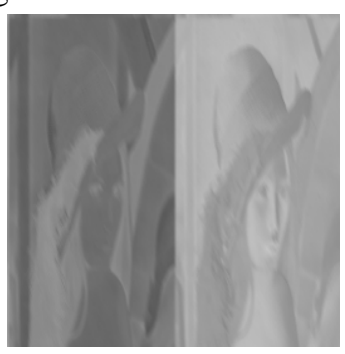

(b) UV plan

Fig.5 Y, UV plane of blurred image

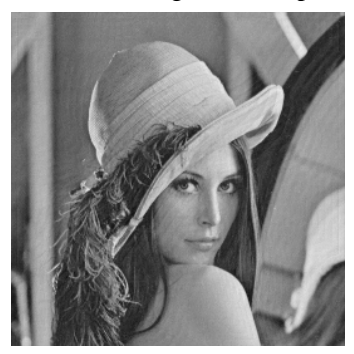

(a)Y plane

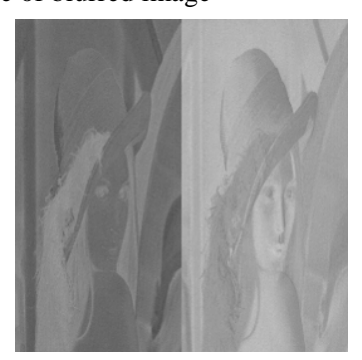

(b) UV plan
Fig.6 Y,UV plane of restored image

\section{ADVANCED COMBINATION}

When the PSF is acquired from the deterministic blur, it will be a good choice to choose the algorithm based on frequency filtering, such as Wiener filter, to finish the restoration. The advanced combination between $\mathrm{Y}$ and $\mathrm{UV}$ plane is based on the Fourier 
frequency processing, and it will be introduced in the following.

\subsection{Symmetry property of FFT}

The fast Fourier transformation is very important algorithm that transforms the image into frequency domain. It is known that the two dimensional Fourier transform can be calculated by applying the transform in one direction and then in the other direction. So the property of two dimensional Fourier transformation can be obtained from the discussion of one dimensional Fourier transform.

$$
F(u)=\sum_{x=0}^{N-1} f(x) W_{N}^{u x}
$$

From (10) it can be seen that if $f(x)$ is a real sequence, the Fourier transform has much symmetry:

$$
F(u)=F^{*}(N-u)
$$

Two dimensional Fourier transform has the same symmetry property too which can be found in $[9,10]$. It is clear that the frequency spectrum of image is symmetry.

\subsection{Advanced combination}

Computing both parts of these conjugates as (11) is inefficient. Some efficient methods are given in. [9, 11] for the compression of Fourier frequency. Based on them, an improved approach for frequency compression is proposed in this paper. First, the $\mathrm{y}_{\mathrm{k}}(\mathrm{x}), \mathrm{u}_{\mathrm{k}}(\mathrm{x})$ are assumed as the $k$ th row in $\mathrm{Y}$ and UV plane respectively. A new, complex sequence, $t_{k}(x)$ is defined by:

$$
t_{k}(x)=y_{k}(x)+i u_{k}(x)
$$

$t_{\mathrm{k}}(\mathrm{x})$ can be easily defined without any data movement by pointing to one row of $\mathrm{Y}$ plane as the real values and one row of UV plane as the imaginary values. $t_{\mathrm{k}}(\mathrm{x})$ can be calculated in half the time it would take to calculate $\mathrm{y}_{\mathrm{k}}(\mathrm{x})$ and $\mathrm{u}_{\mathrm{k}}(\mathrm{x})$ individually. Because the Fourier transform is linear, we get

$$
T_{k}(u)=Y_{k}(u)+i U_{k}(u)
$$

But the $T_{k}(u)$ is not useful to us. We need to extract the $Y_{k}(u)$ and $U_{k}(u)$ from $T_{k}(u)$. We know that
$\mathrm{Y}_{\mathrm{k}}(\mathrm{u})$ and $\mathrm{U}_{\mathrm{k}}(\mathrm{u})$ display the conjugate symmetry discussed above because $\mathrm{y}_{\mathrm{k}}(\mathrm{x})$ and $\mathrm{u}_{\mathrm{k}}(\mathrm{x})$ are real. Hence,

$$
\begin{aligned}
T_{k}(N-u)= & Y_{k}(N-u)+i U_{k}(N-u) \\
& =Y_{k}^{*}(u)+i U_{k}^{*}(u)
\end{aligned}
$$

Combining (13) and (14), we find that

$$
\begin{aligned}
Y_{k}(u)_{R} & =\frac{1}{2}\left[T(u)_{R}+T(N-u)_{R}\right] \\
Y_{k}(u)_{I} & =\frac{1}{2}\left[T(u)_{I}-T(N-u)_{I}\right] \\
U_{k}(u)_{R} & =\frac{1}{2}\left[T(u)_{I}+T(N-u)_{I}\right] \\
U_{k}(u)_{I} & =\frac{1}{2}\left[T(N-u)_{R}-T(u)_{R}\right]
\end{aligned}
$$

where the R and I subscripts stand, respectively, for the real and imaginary components of a complex number. From (15) it can be seen that the extract procedure of $\mathrm{Y}_{\mathrm{k}}(\mathrm{u})$ and $\mathrm{U}_{\mathrm{k}}(\mathrm{u})$ is just concerned about some simple arithmetical operations, such as addition, subtraction and bitwise.

\subsection{The data configuration and 2D FFT}

The discussion in section 5.2 is the Fourier transform for one dimensional sequence, but the images are two dimensional, so we move the focus from one dimensional Fourier transform to two dimensional Fourier transform in this section.

There are two special cases that when $\mathrm{u}=0$ or $\mathrm{u}=\mathrm{N} / 2, \quad \mathrm{~T}_{\mathrm{k}}(\mathrm{u})=\mathrm{T}_{\mathrm{k}}(\mathrm{N}-\mathrm{u})$. It falls out from (15) that $\mathrm{Y}_{\mathrm{k}}(0)_{\mathrm{R}}=\mathrm{T}_{\mathrm{k}}(0)_{\mathrm{R}} / 2, \mathrm{Y}_{\mathrm{k}}(0)_{\mathrm{I}}=0, \mathrm{U}_{\mathrm{k}}(0)_{\mathrm{R}}=\mathrm{T}_{\mathrm{k}}(0)_{\mathrm{I}}, / 2, \mathrm{U}_{\mathrm{k}}(0)_{\mathrm{I}}=0$. Likewise for values of $Y_{k}(N / 2)$ and $U_{k}(N / 2)$. In the Fourier spectra with $\mathrm{N}$ complex points, $\mathrm{N} / 2-1$ complex values are conjugates of values, and the imaginary part of values at indices 0 and N/2 are always zero. This leaves us with only $\mathrm{N} / 2$ complex values we need to store in our array. Hence, $\mathrm{Y}_{\mathrm{k}}(\mathrm{u})$ and $\mathrm{U}_{\mathrm{k}}(\mathrm{u})$ can be efficiently stored in an array the same size as $t_{\mathrm{k}}(\mathrm{x})$ sequence. Fig.7-a is the storage of $t_{\mathrm{k}}(\mathrm{x})$, Fig.7-b shows how we efficiently packed the values for $\mathrm{Y}_{k}(\mathrm{u})$ and $\mathrm{U}_{\mathrm{k}}(\mathrm{u})$. 


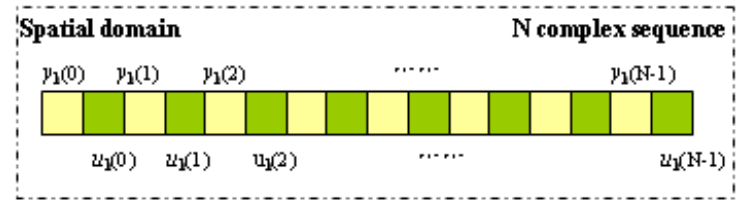

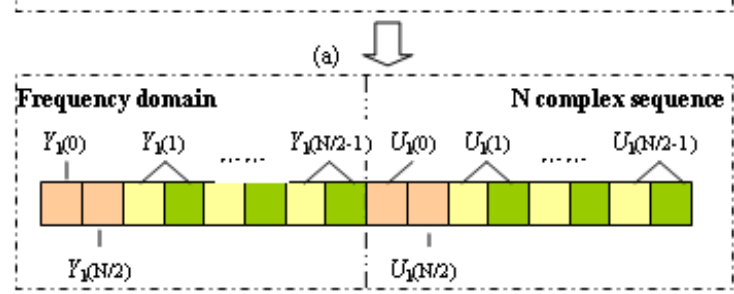

(b) உ

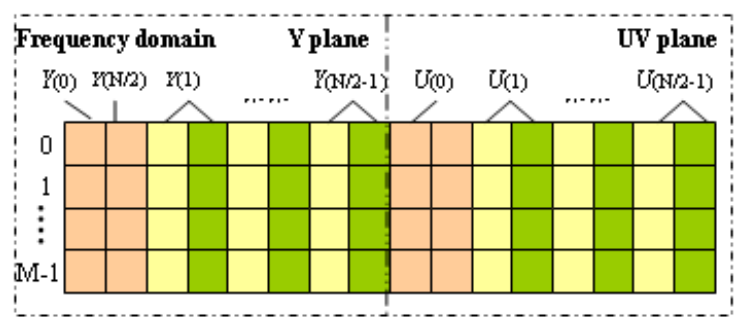

(c) 凸

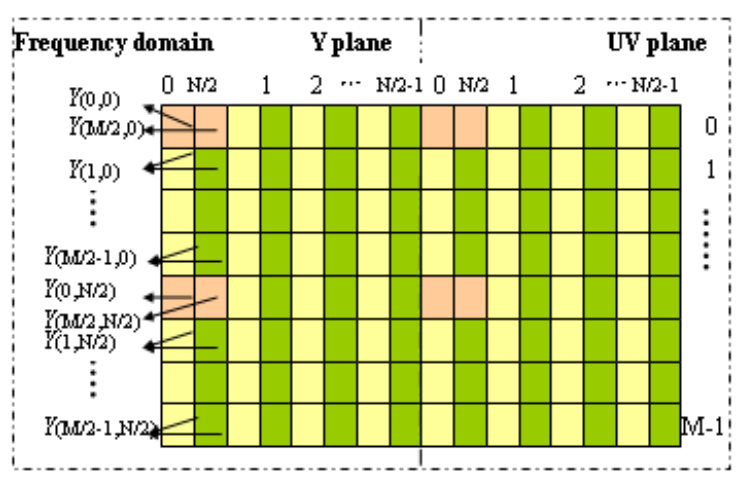

(d)

$\square$ special part $\square$ Real part $\square$ Imaginary part

Fig.7 Organization of YUV data during FFT

After performing the 1D FFT on the rows of $\mathrm{Y}$ and UV plane and packing them as show in Fig.7-c, we need to perform the FFT on the columns. Four of the columns marked as special part in Fig.7-c contain real numbers that are real part of values at indices 0 and N/2 of each rows of $\mathrm{Y}$ and UV plane. We can pair them up and perform the FFT on them together with the method described above. The rest of the columns are properly paired as sequences of complex numbers. We perform a traditional complex to complex FFT on these sequences. Fig.7-d shows how the final result is packed.

Computing the two dimensional FFT by combining $\mathrm{Y}$ and UV plane together as described above takes about half the time as computing the FFT on them directly and separately.

\section{EXPERIMENTS AND RESULTS}

In the experiment, the motion blurred color image is converted into YUV color space, and the $\mathrm{Y}, \mathrm{U}$ and $\mathrm{V}$ plane are combined as described above. Under the described FFT performing, the frequency spectrum of $Y$, UV plane and their PSF are generated. The Wiener filter is applied to restore the motion blurred color image based on the frequency spectrums. With the technology of general purpose $\mathrm{GPU}^{[12]}$, the proposed algorithm is parallelly executed on GPU. The GPU we used in the experiment is nVidia GeForce8800GTS (512M).
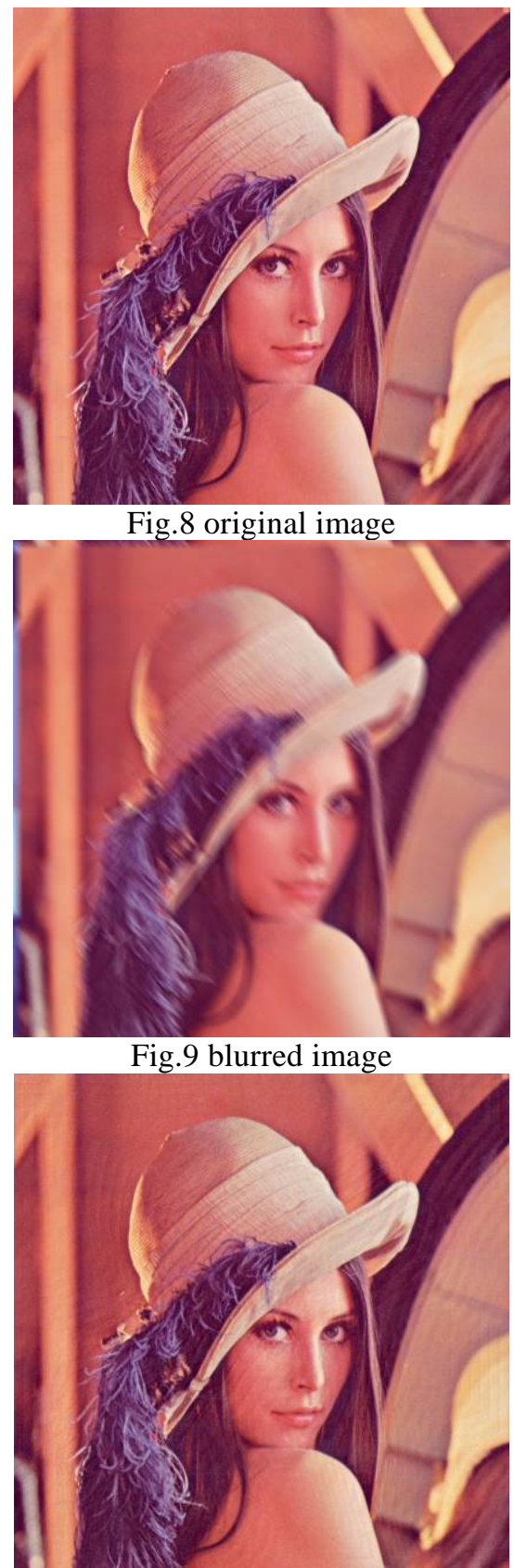

Fig.10 restored image 
The lab test image is color Lena (512x512) shown in Fig.8. Fig.9 is the motion blurred image of Fig.8, and Fig.10 is the filtered image of Fig.9 by Wiener filter based on the PSF expression (9). Fig. 5, 6 shown in section 4.2 are the $\mathrm{Y}, \mathrm{U}, \mathrm{V}$ plane of Fig. 9, 10 respectively. The estimation of $\mathrm{Y}, \mathrm{U}$, and $\mathrm{V}$ plane of Fig.10 is shown in Tab.1.

Tab.1 MSE and PSNR of Y, U, V value of Lena

\begin{tabular}{|l|l|l|l|l|l|}
\hline Image & MSE/PSNR & $\mathrm{Y}$ & $\mathrm{U}$ & $\mathrm{V}$ & Ave. \\
\hline \multirow{2}{*}{ Lena } & MSE & 29.29 & 16.00 & 16.58 & 20.62 \\
\cline { 2 - 6 } & PSNR & 33.46 & 36.09 & 35.94 & 35.16 \\
\hline
\end{tabular}

The TV monitors are working in RGB color space, so the estimation results of $\mathrm{R}, \mathrm{G}$, and $\mathrm{B}$ of Fig.10 are more important to HVS, and they are shown in Tab.2. We also use the traditional algorithm, filter the R, G, B plan directly by wiener, to restore the color Lena and the contrast estimation results are shown in Tab.2 too. The estimation data in Tab.1and Tab.2 indicate that there is a little image degradation when color converted between YUV422 and RGB888, but the restored result is acceptable.

Tab.2 PSNR of R, G, B value of Lena by different restored algorithms

\begin{tabular}{|c|c|c|c|c|l}
\hline Parameter & Algorithm & $\mathrm{R}$ & $\mathrm{G}$ & $\mathrm{B}$ & Ave. \\
\hline \multirow{2}{*}{ PSNR } & proposed & 31.35 & 32.91 & 30.08 & 31.45 \\
\cline { 2 - 6 } & Traditional. & 30.90 & 31.57 & 34.90 & 32.46 \\
\hline
\end{tabular}

The purpose of Y, U, and V combination is for fast restoration of motion blurred color video. We test the restoration efficiency of proposed algorithm and traditional algorithm separately. The test content includes the restoration with different image sizes and the restoration with different pixel depth. The both algorithm are optimized by GPGPU ${ }^{[13]}$ and all the tests are operated on nVidia GeForce8800GTS. The test results are listed in Tab.3. The data in Tab.3 show that the proposed algorithm can restore the blurred color images in the same time as it is needed to restore the same size gray level images by the traditional algorithm, and the results also reveal that the proposed algorithm is about 3X speed of the traditional algorithm.

Tab.3 result of time test (unit: ms)

\begin{tabular}{|c|c|c|c|c|}
\hline Algorithm & $\begin{array}{c}\text { pixel } \\
\text { depth }\end{array}$ & $256 \times 256$ & $512 \times 512$ & $1024 \times 1024$ \\
\hline Proposed. & $24 b i t$ & 0.475 & 2.08 & 7.95 \\
Traditional. & 8bit & 0.467 & 2.05 & 7.93 \\
Traditional. & 24bit & 1.378 & 6.16 & 23.71 \\
\hline
\end{tabular}

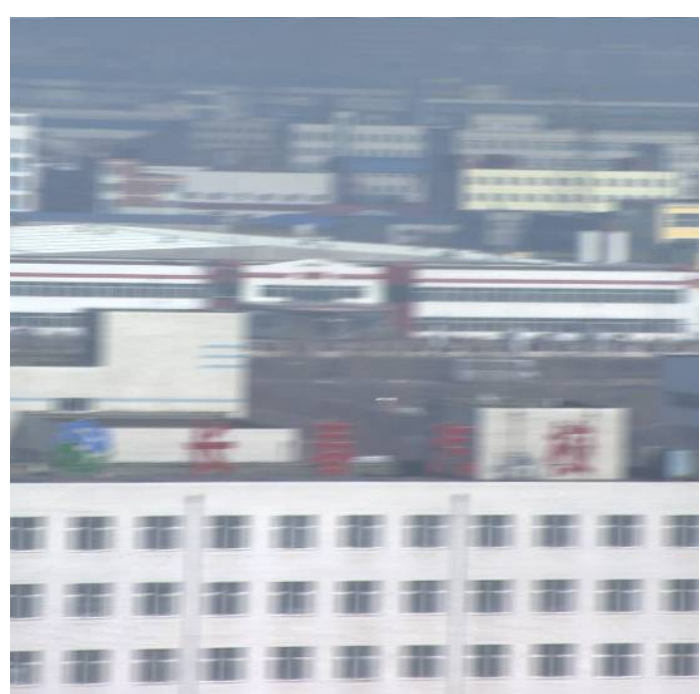

Fig.11 real motion blurred image (1024x1024 24 bit)

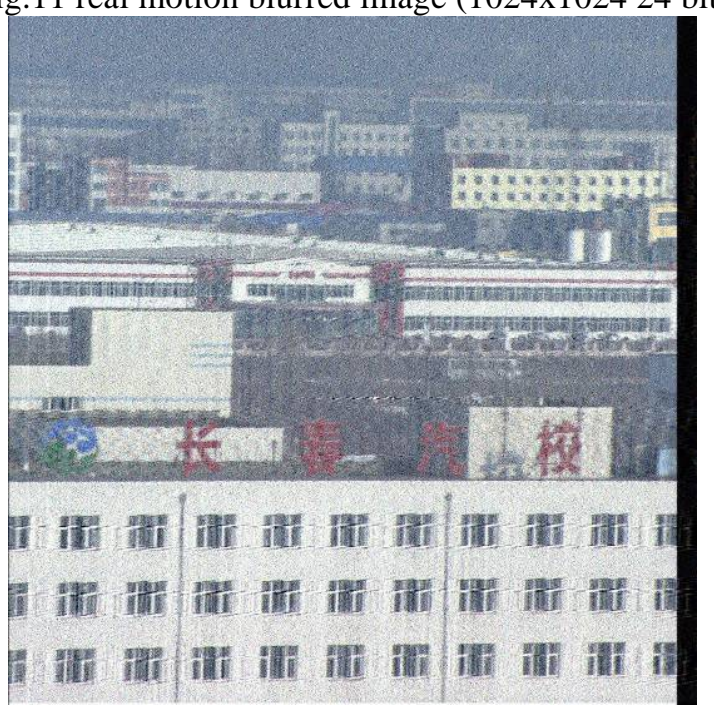

Fig.12 restored image (1024x1024 24 bit)

To make the experiment more complete, we capture the real motion blurred color video and try to use the proposed algorithm to restore the blurred frames in real time. Before the proposed algorithm operates, we apply the windowing technique ${ }^{[14,15]}$ to preprocess the edge error of the real blurred frame. The real motion blurred color image is shown in Fig.11. Fig.12 is the restored image of Fig.11 using the proposed algorithm. Notice that the image sharpness is greatly enhanced. Considered the image transfer time, the average restoration time for one frame, $1024 \times 1024$ 24bit, is 18 ms on GPU.

\section{CONCLUSIONS}

In this paper a fast restoration algorithm for color images is described which shows a good performance for real time processing. The transform of image format 
from RGB888 to YUV422 can reduce about $1 / 3$ computational requirement, and the procedure of $\mathrm{Y}, \mathrm{U}$, $\mathrm{V}$ plane combination, composed Fourier spectra production and composed Fourier spectra separation can cut half calculated amount again. Taking advantage of these procedures, the proposed algorithm attains about $3 \mathrm{X}$ the efficiency of the traditional algorithm. The experiment results show that the proposed algorithm can

\section{REFERENCES}

[1]H. H. Jhang, Using Support Vector Committee Machine for Face Tracking Based on Adaptive Color Space Switching, Graduate school of electronic engineering national yunlin university of science \& technoloty, 2006

[2]S Süsstrunk, R Buckley, S Swen, Standard RGB Color Spaces, Proc. IS\&T/SID 7th Color Imaging Conference, 1999

[3]H. C. Andrews and B.R. Hunt, Digital Image Restoration. Englewood Cliffs, NJ: Prentice-Hall, 1977.

[4]M. R. Banham, A. K. Katsaggelgs, ”Digital image restoration,” IEEE signal proc. mag.. 1997 ,March: 24-41

[5]P. Jia, H. Sun, B. Zhang, "Restoration of motionblurred aerial image," Optics and Precision Engineering, 2006, 14(4) : 697-703

[6]D Tschumperle, $\mathrm{R}$ Deriche, "Constrained and unconstrained PDEs for vector image restoration”, Scandinavian Conference on Image Analysis, Bergen, Norway, June 2001.

[7]I. E. G. Richardson. Video Codec Design: Developing Image and Video Compression Systems. John Wiley \& Sons LTD. 2002

[8]D Chai, A Bouzerdoum, "A Bayesian approach to skin color classification in YCbCr colorspace," TENCON 2000. Proceedings, 2000 vol.2: 421-424

[9]Steven W. Smith, The Scientist and Engineer's Guide to Digital Signal Processing. California Technical Publishing,(1997)

[10]S. Li, B. Zhang, H. Sun "Real-time restoration using real discrete Fourier transform for aerial E-O imaging system," Optics and Precision Engineering, 2007, 15(8) : 1287-1292

[11]K. Moreland, E. Angel, "The FFT on a GPU," In SIGGRAPH/Eurographics Workshop on Graphics Hardware 2003 Proceedings, 2003 July: 112-119

[12]M. Pharr, R. Fernando GPU Gems2. AddisonWesley Professional,March 13, 2005

[13]S. Li, B. Zhang, H.Sun. "Parallel restoration for motion-blurred aerial image. "Optics and Precision Engineering, 2009, 17(1):225-230

[14]Tan K C, Lim H, Tan B T G. Restoration of realworld motion-blurred images, CVGIP, 1991, 53: 291-299. restore a $1024 \times 1024$ color image in $7.95 \mathrm{~ms}$ with $\mathrm{nVidia}$ GeForce8800GTS (512M)

\section{ACKNOWLEDGMENT}

This work was supported by the advanced research foundation for national defense of China.

[15]Tan K C, Lim H, Tan B T G. Windowing techniques for image restoration. CVGIP, 1991, 53: 491-500.

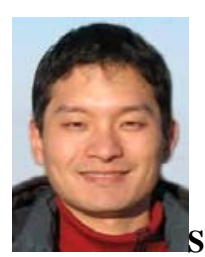
information engineering from Zhejiang University, China, in 2003, and his MS degree in optical engineering from Changchun Institute of Optics, Fine Mechanics and Physics, Chinese Academy of Sciences, in 2007, where he is currently pursuing his $\mathrm{PhD}$ degree in optical engineering. His current research interests include image restoration, real-time image processing, and general purpose GPU.

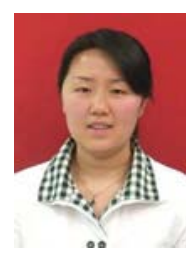

Yuping Feng received her BS degree in electronic and information engineering from Changchun University of Science and Technology, in 2005, and her MS degree in optical engineering from Changchun Institute of Optics, Fine Mechanics and Physics, Chinese Academy of Sciences, in 2007, where she is currently studying for a $\mathrm{PhD}$ degree in optical engineering. Her current research interests are image matching, image mosaic, and image fusion. 
Bao Zhang is a professor at the Changchun Institute of Optics, Fine Mechanics and Physics, Chinese Academy of Sciences. He received his BS degree in optical instrumentation, MS degree in fine instrumentation, and $\mathrm{PhD}$ degree in mechanical engineering from Changchun Institute of Optics, Fine Mechanics and Physics, Chinese Academy of Sciences, in 1989, 1994, and 2004, respectively. His major research interest is the research and manufacture of opto-electronic reconnaissance platforms. $\mathrm{He}$ is a Member of the Chinese Optics Society.

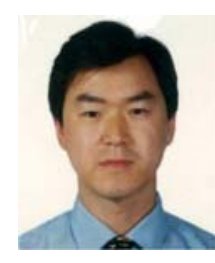

Hui Sun received his BS degree in mathematic computing from Jilin University in 1985. He has been a professor at Changchun Institute of Optics, Fine Mechanics and Physics, Chinese Academy of Sciences. He is devoted to the design and development of computer software. His current research interests include digital image processing, analysis, and computer simulation. 